\title{
Médiévales
}

Langues, Textes, Histoire

61 | automne 2011

La chair des émotions

\section{Du vilain au paysan sur la scène littéraire du $\mathrm{XIII}^{\mathrm{e}}$ siècle}

From Villein to Peasant in the Literature of the 13th Century

\section{Marie-Thérèse Lorcin}

\section{(2)enEdition}

Journals

Édition électronique

URL : https://journals.openedition.org/medievales/6551

DOI : 10.4000/medievales.6551

ISSN : $1777-5892$

\section{Éditeur}

Presses universitaires de Vincennes

\section{Édition imprimée}

Date de publication : 20 décembre 2011

Pagination : 163-186

ISBN : 978-2-84292-337-2

ISSN : 0751-2708

Référence électronique

Marie-Thérèse Lorcin, " Du vilain au paysan sur la scène littéraire du xı॥ siècle », Médiévales [En ligne], 61 | automne 2011, mis en ligne le 29 juin 2012, consulté le 22 avril 2022. URL : http://

journals.openedition.org/medievales/6551; DOI : https://doi.org/10.4000/medievales.6551 


\section{Marie-Thérèse LORCIN}

\section{DU VILAIN AU PAYSAN SUR LA SCÈNE LITTÉRAIRE DU XIII SIÈCLE}

L'image que donne du paysan la littérature française du Moyen Âge continue d'intriguer. Le stéréotype du vilain, incarnation de la bassesse, se rencontre dans les genres littéraires les plus divers, suscitant maints commentaires.

Dès 1965, Jacques Le Goff remarquait qu'au moment même où la civilisation de la Romania se ruralisait, le paysan disparaissait de l'écrit. Dès les $\mathrm{V}^{\mathrm{e}}$ et $\mathrm{VI}^{\mathrm{e}}$ siècles, il faut le chercher sous le masque du païen, du pauvre, de l'illettré et des classes dangereuses. Lorsqu'il réapparaît, il conserve les traits péjoratifs attribués précédemment, si bien que le vilain ignorant et vicieux a l'allure d'un «Caliban médiéval», expression qui fit fortune ${ }^{1}$.

$\mathrm{Au}$ fil du temps, la situation devint naturellement plus complexe et fournit à nombre d'historiens et littéraires un thème de recherche. En 1976, Jean Batany explorait le vaste champ des écrits moralisants et didactiques, tant en latin qu'en français et italien, pour montrer comment évolue, des Bucoliques à la Renaissance, l'idée du «bonheur des paysans » ${ }^{2}$. Dix ans plus tard, Michel Zink tira des pastourelles et des premières pièces de théâtre le portrait plaisant de jeunes paysans et paysannes gais, insouciants, volontiers frondeurs à l'égard de l'aristocrate égaré dans les pâtures ${ }^{3}$. Jean Dufournet montrait peu après que les

1. J. LE GoFf, «Les paysans et le monde rural dans la littérature du haut Moyen Âge ( $\mathrm{v}^{\mathrm{e}}$ $\mathrm{VI}^{\mathrm{e}}$ siècles)», dans Agricoltura e mondo rurale in Occidente nell'alto medioevo : 22-28 aprile 1965 (Settimane di studio del Centro italiano sull'alto medioevo, 13), Spolète, 1966, p. 723-745.

2. J. BAtAnY, «Le bonheur des paysans des Géorgiques au bas Moyen Âge», dans R. CHEVALLIER éd., Présence de Virgile, actes du colloque des 9-12 décembre 1976, Paris, 1978 (Caesarodunum, $\mathrm{n}^{\mathrm{o}}$ spécial 13 bis), p. 233-248.

3. M. ZinK, «La suffisance du paysan dans la littérature française du Moyen Âge », dans W. Hirdt éd., Der Bauer im Wandel der Zeit, Bonn, 1986, p. 40-48. 
activités du vilain Liétard mis en scène dans la branche $\mathrm{X}$ du Roman de Renart correspondent fort bien à l'économie rurale de la Brie à la fin du XII ${ }^{\mathrm{e}}$ siècle ${ }^{4}$. J'ai moi-même esquissé, à partir des fabliaux, le personnage d'un chef de feu rural qui a des terres, une mesnie, des responsabilités, et qui fait preuve de bon sens et de générosité ${ }^{5}$. Les recueils de proverbes du Moyen Âge central contiennent à la fois des sentences on ne peut plus méprisables à l'égard du vilain et la formule qui sera reprise partout - «ci dist li vilain »-, qui fait de celui-ci le dépositaire de la sagesse des nations ${ }^{6}$.

On pourrait rappeler ici bien d'autres travaux. Évoquons pour finir un des plus récents qui, de prime abord, semble n'avoir aucun lien avec le sujet. Ghislain Brunel attire l'attention sur les chartes de franchises et contrats agraires de la moitié septentrionale de la France dont le latin «se perle de français». Des termes techniques s'introduisent au cours des $\mathrm{XII}^{\mathrm{e}}$ et XIII ${ }^{\mathrm{e}}$ siècles, tel «operas que vulgariter manuovres appellantur ». L'auteur conclut:

À l'évidence, ces mots de langue vulgaire claquent d'un bruit différent au milieu du latin; il suffit de les lire à haute voix pour en ressentir le timbre particulier. Fort probablement, l'emploi des mots français provient d'une réclamation de la communauté paysanne intéressée. Entendre, lors de la lecture de l'acte, les mots exacts du litige, non pas excubias ou pernoctationes, mais les gistes, facilitait l'accord et la confiance réciproques ${ }^{7}$.

Nous voici loin d'Aucassin et Nicolette et du Vilain Mire... Pourtant cette recherche met en valeur un trait curieux. Dans les textes littéraires, le vilain le plus crasseux s'exprime aussi élégamment que le clerc ou le bourgeois. Le vilain Liétard parle comme Renart et Ysengrin qui sont des seigneurs. On ne voit pas le «parler paysan». Les satiristes d'alors montent en épingle la façon dont Anglais et Bretons baragouinent le français, mais n'attribuent jamais au vilain un charabia. Comme le remarque Serge Lusignan, «les auteurs médiévaux voient uniquement la différence dans les parlers comme indicateur d'un lieu d'origine différent et jamais comme un marqueur d'origine sociale ${ }^{8} \gg$. Les poètes n'emploient pas ce moyen d'exclure le paysan du reste de la société.

4. J. Dufournet, «Portrait d'un paysan du Moyen Âge: le vilain Liétard», dans ID. éd., Le Goupil et le Paysan: Roman de Renart, branche X, Paris, 1990, p. 57-105.

5. M.-T. LoRCin, Façons de sentir et de penser : les fabliaux français, Paris, 1979.

6. EAD., Les Recueils de proverbes français (1160-1490). Sagesse des nations et langue de bois, Paris, 2011.

7. G. BRUNEL, «Entre concessio et conventio. Le vocabulaire du prélèvement seigneurial en France du Nord (XII-XIve siècle)», dans M. Bourin, P. Martínez Sopena éd., Pour une anthropologie du prélèvement seigneurial dans les campagnes médiévales : $X I^{e}-X I v^{e}$ siècle, réalités et représentations paysannes, Paris, 2004, p. 109-135.

8. Je remercie Serge Lusignan de sa réponse écrite à ma question sur le sujet. 
Tout cela révèle, à côté du Caliban médiéval, un autre paysan, un paysan qui fait figure d'homme ordinaire. Il ne surgit pas tout d'une pièce mais se constitue peu à peu. Il ne fait pas disparaître le vilain laid, bête et envieux, objet de mépris qui poursuit sa carrière dans les contes et ailleurs. Parfois les deux figures se mêlent et le paysan prend comme Janus un double visage.

Le but de ces quelques pages n'est pas de faire le tour complet d'un sujet qui se révèle fort vaste. Ma quête est limitée dans le temps, dans l'espace, dans le choix des sources et dans leur questionnement.

La période choisie est comprise entre le milieu du XII ${ }^{e}$ siècle et le début du XIV ${ }^{e}$ siècle. C'est la belle époque où la France tire profit de l'essor entamé antérieurement dans tous les domaines. Elle connaît comme tout l'Occident une poussée urbaine telle qu'il ne s'en était pas produit depuis la chute de Rome. La surface cultivée atteint un de ses sommets historiques. Les denrées agricoles produites écartent le spectre des grandes famines, l'aisance progresse et les franchises s'obtiennent plus facilement. Toutefois la campagne ne peut nourrir et donner du travail à tous les jeunes. Beaucoup partent chercher fortune en ville. Parmi les garçons fréquentant les écoles urbaines au XIII ${ }^{\mathrm{e}}$ siècle se trouve maint fils de paysan, comme le remarque Rutebeuf dans le Dit de l'université de Paris :

Li filz d'un povre païsant

Vanrra a Paris por apanre;

Quanque ces peres porra panre

En un arpans ou II de terre

Por pris et por honeur conquerre

Baillera trestout a son fil

Et il en remaint a escil ${ }^{9}$.

Au début du XIV siècle, l'ambiance devient moins favorable. Famine, peste et guerre ne tardent pas à se répandre. Les soulèvements suivent, en ville puis à la campagne. Après la Jacquerie de 1358, à l'image du vilain qui fait rire s'ajoute celle du «Jacques» qui fait peur. Les conditions de l'étude ne sont plus les mêmes ${ }^{10}$.

C'est dans le Bassin parisien que la prospérité du long XIII ${ }^{\mathrm{e}}$ siècle est la plus visible. Ignorant la crise albigeoise, la moitié septentrionale de la France jouit longtemps de la paix que font régner les souverains capétiens. C'est le domaine de la langue d'oïl et c'est grosso modo la zone où furent composés les fabliaux. Les variétés régionales de la langue permettent d'attribuer tel conte

9. Rutebeuf, Euvres complètes, éd. M. ZinK, 2 vol., Paris, 1989-1990.

10. M.-T. Lorcin, «La Jacquerie de 1358 : un mal parmi d'autres, un mot qui répand la terreur», Histoire médiévale, 61 (2005), p. 27-33. 
à la Normandie, tel autre à la Champagne, le plus grand nombre à «la région picardisante $»^{11}$.

Les sources choisies appartiennent en majorité aux «genres secondaires». Les épopées et romans en effet ne font aux paysans qu'une place minime. Dans le Roman de Renart lui-même, les branches X et XVI sont seules à donner aux vilains plus qu'une dizaine de lignes. La place de ceux-ci est plus grande dans les recueils de miracles en français, en particulier ceux de Notre-Dame de Chartres ${ }^{12}$. La source principale reste le corpus des fabliaux parce qu'il accorde aux paysans une place inusitée et les présente sous les jours les plus variés, du plus méprisable au plus digne. À cela s'ajoutent de courtes pièces satiriques publiées jadis par Edmond Faral dont certaines sont très proches des fabliaux (L'Eschacier par exemple $)^{13}$. On ne peut oublier bien sûr des jeux mettant en scène bergers et bergères tel celui de Robin et Marion ${ }^{14}$. Il faut aussi inclure les recueils de proverbes antérieurs à 1340 dont j'ai établi la typologie. Enfin et surtout, Rutebeuf qui non seulement a composé des fabliaux mais qui glisse fréquemment dans ses poèmes des allusions à l'actualité.

À travers la masse hétéroclite des sources, deux pistes de recherche seront suivies successivement. La première est l'étude du vocabulaire. Plusieurs termes peuvent servir à désigner le paysan et l'auteur ne choisit pas au hasard. Inversement, le mot «vilain» a plusieurs sens: il s'applique au paysan, il peut aussi être une apostrophe injurieuse jetée à des personnages qui ne sont point paysans. Fondamentale, l'étude des thèmes est plus délicate. Il est toujours risqué de traiter les textes littéraires comme des documents. Passe encore d'y puiser, comme dans l'iconographie, des renseignements sur les objets de la vie quotidienne et la manière de s'en servir... Chercher dans les mêmes sources le reflet de relations sociales, des façons de sentir et de penser, pour certains, frise l'hérésie.

Ma démarche consistera modestement à examiner une fois de plus le stéréotype du vilain et à montrer comment les textes du XIII ${ }^{\mathrm{e}}$ siècle en corrigent l'absolutisme en offrant un modèle différent qui est une nouveauté. Stéréotyper est un procédé de base de la perception humaine. C'est, nous disent les sociologues,

11. W. Noomen et N. Van den BoogaArd éd., Nouveau recueil complet des fabliaux (ci-dessous abrégé N.R.C.F.), 10 vol., Assen, 1983-1998.

12. Jean le Marchant, Miracles de Notre-Dame de Chartres, éd. P. Kunstmann, OttawaChartres, 1973.

13. E. FARAL, «Des vilains ou des XXIII manières de vilains», Romania, 48 (1922), p. 243264; ID., Mimes français du XIII siècle, contribution à l'histoire du théâtre comique du Moyen Âge, Genève, 1973, qui contient «Le privilège aus Bretons », p. 3-28, «La paix aus Englois », p. 31-35, «Les deux bourdeurs ribauds», p. 83-111.

14. Adam le Bossu, «Le jeu de Robin et Marion», dans A. Pauphilet éd., Jeux et sapience du Moyen Âge, Paris, 1941, p. 159-201. 
le réflexe positif de l'individu à l'égard du groupe dont il fait partie, en même temps que le réflexe négatif qu'il éprouve à l'égard des autres groupes. À partir de vagues similitudes entre le physique et le moral est construite une image rigide qui se suffit à elle-même. C'est pour l'individu un facteur de sécurisation.

Comme les stéréotypes tendent à s'exprimer par des formes proches de la caricature, les plus vigoureusement dessinés se trouvent dans les textes comiques et satiriques. Tels sont dans les fabliaux les stéréotypes du prêtre, du vilain, de la femme. Le stéréotype est en même temps un procédé commode, pour ne pas dire économique, qui évite de décrire et d'expliquer. Un mot suffit, même si le conteur ne croit pas à la véracité des préjugés qu'il évoque. Toutefois le stéréotype du vilain est moins cohérent que celui du prêtre analysé par Daron Burrows ${ }^{15}$. Les canons conciliaires, les mandements épiscopaux, permettent de dresser le portrait idéal du prêtre auquel opposer ensuite les composantes du stéréotype tirées des fabliaux. Bien qu'il existe des sermons adressés aux paysans, surtout en tant que décimables, il faudrait beaucoup d'imagination pour dresser à partir de textes normatifs le portrait idéal du paysan.

Dans le corpus des textes utilisés, les traits favorables côtoient les critiques à l'égard du vilain, comme il a été rappelé plus haut. Beaucoup de clercs habiles à versifier sont sans doute d'origine rurale, ce qui contribue à expliquer leur diversité de ton. Or il serait simpliste de traiter le matériau comme sont pesées les âmes lors du Jugement dernier. Le vilain courtois et hospitalier n'est pas nécessairement plus « réel» que le vilain brutal et envieux. La quête s'emploie à relever les moyens qu'utilisent les écrivains pour nuancer le portrait du paysan. Quel statut, quel comportement, quels traits, physiques ou moraux, doit-il avoir pour paraître aussi normal qu'un autre personnage?

\section{Les mots pour le dire}

Il existait au XIII ${ }^{\mathrm{e}}$ siècle plusieurs termes pour désigner le paysan. Dans les Miracles de Notre-Dame de Chartres, rimés par Jean le Marchant entre 1252 et 1262, «païsant» et «vilein » sont employés comme synonymes dans les deux récits qui concernent les paysans. Le miracle II est l'histoire d'un valet nommé Benoit $^{16}$ :

Cil Beneoit, com preuz et sage

A un païsant d'un vilage

S'aloua a ses ovres faire (v. 6-10)

15. D. Burrows, The Stereotype of the Priest in the Old French Fabliaux. Anticlerical Satire and Lay Identity, Berne, 2005.

16. Jean le Marchant, II, v. 6-10. 
Son employeur, hélas, le fit travailler un jour de fête.

Le vilein fel, de pute afeire (v. 11)

Le thème est le même dans le miracle XXVI «dou vilein qui saiet s'avoine a la feste de saint Germain» :

Si avint a un païsant. (v. 13)

Cil vilein es chans laboroit. (v. 17)

Le terme «païsant» se rencontre aussi dans Le Roman de la Rose ${ }^{17}$. Rutebeuf l'utilise au moins deux fois comme nous le verrons plus loin. En revanche il est rare dans les contes. Parmi les 127 fabliaux du Nouveau recueil complet des fabliaux, quatre seulement en usent:

Les Quatre Prestres : «li païsant», trois occurrences, premier tiers du XIII siècle, Normandie.

Constant du Hamel: «son paysan », une occurrence, première moitié du XIII ${ }^{\mathrm{e}}$ siècle, Picardie.

Le Vilain Mire: «le païsant», une occurrence, seconde moitié du XIII ${ }^{\mathrm{e}}$ siècle, Île-de-France.

La Fame qui cunquie: « uns païssans », trois occurrences, XIII ${ }^{\mathrm{e}}$ siècle (?), origine incertaine.

Peut-être le terme est-il jugé trop savant pour un genre familier qui ne recule point devant le trivial et l'obscène. Toujours est-il que le mot courant est «vilain », et non «païsant». L'usage en est si répandu que l'auteur du conte juge inutile de s'expliquer. Le décor et l'action confirment suffisamment, même pour le lecteur actuel, qu'il s'agit bien du paysan.

Fabliaux où «vilain» désigne un paysan:

Début du XIII ${ }^{\mathrm{e}}$ siècle: sept contes, dont six de Jean Bodel.

$1^{\text {re }}$ moitié du XIII ${ }^{\mathrm{e}}$ siècle: onze contes.

Milieu du XIII ${ }^{\mathrm{e}}$ siècle: deux contes, dont un de Rutebeuf.

$2^{\mathrm{e}}$ moitié du XIII ${ }^{\mathrm{e}}$ siècle: quatre contes, dont trois de Gautier le Leu.

$\mathrm{XIII}$ e siècle: six contes.

Au temps de Jean Bodel, qui compose des fabliaux vers 1190-1200, l'identité vilain = paysan semble aller de soi. Et ce vilain n'est pas nécessairement un être hideux, méprisable et stupide. Jean Bodel met en scène des paysans sympathiques. Ils font sourire par leur naïveté, leur générosité, les commentaires qu'ils font de la situation. Ainsi le brave homme qui propose à sa femme de donner au prêtre

17. Guillaume de Loris et Jean de Meung, Le Roman de la Rose, éd. F. Lecoy, 3 vol., Paris, 1965-1970, v. 18869. 
leur unique vache ne rencontre aucune opposition. Il ajoute cependant comme argument supplémentaire :

Aussi rent ele petit lait! ${ }^{18}$

Ébloui par la bonté du Créateur qui leur rend deux vaches au lieu d'une, le vilain remarque:

Petit sera nostre toitiaus!

Ces vilains sont les uns pauvres, comme dans Les Deus Chevaus et Le Vilain de Bailluel, les autres fort à l'aise. Gombert, qui héberge sans défiance deux jeunes clercs entreprenants, s'évertue à installer ses hôtes confortablement dans un bon lit non loin du foyer, et toute la maisonnée au préalable a savouré un repas réconfortant ${ }^{19}$.

Cependant employer le mot «vilain» est délicat. Dans une dizaine de contes, ce mot est une injure qui vient facilement à la bouche d'un personnage irrité, et celui auquel elle s'adresse n'est pas un paysan. Situation qui apparaît dans le plus ancien des fabliaux. La Male Honte, dans sa première version, est un conte antérieur au milieu du XII ${ }^{\mathrm{e}}$ siècle ${ }^{20}$. Le personnage dévoué et respectueux du droit est «uns preudons » que le roi d'Angleterre en colère apostrophe ainsi :

Vilain, dist il, tu me mesdiz. (v. 40)

Il en est de même par la suite. Celui qui s'obstine à présenter au roi la malle qui contient l'héritage du défunt nommé Honte est toujours appelé «li preudon», mais le roi, qui se croit offensé, l'interpelle chaque fois par le terme «vilain», même lorsqu'à la fin du conte, le malentendu dissipé, il lui rend justice. Aussi le poète conclut-il en blâmant le «mavès seignor» qui attire la honte (réelle) sur son peuple...

Ce type de situation se rencontre plus d'une fois. Le personnage injurié peut être un artisan, un commerçant, un riche bourgeois. Dans Sire Hain et Dame Anieuse, l'homme est un couturier qui gagne bien sa vie.

Sire Hain savoit bon mestier

Quar il savoit bien rafetier

Les coteles et les mantiaus ${ }^{21}$.

L'auteur le désigne toujours comme «sire Hains » mais son épouse, une mégère non apprivoisée, le traite de vilain (v. 174, 240), voire pis :

18. N.R.C.F., V, 40, De Brunain la vache au prestre, v. 17.

19. N.R.C.F., IV, 40.

20. N.R.C.F., V, 43.

21. N.R.C.F., II, 5, v. 7-9. 
Filz a putain, vilains pullenz. (v. 230)

Elle change de langage une fois chue dans le bac à lessive dont elle ne peut sortir seule, trouvaille reprise plus tard dans la farce du Cuvier.

Le qualificatif injurieux n'est pas toujours lancé à la face d'autrui. Il peut être proféré par l'amant en l'absence du mari. Dans Les Braies au Cordelier, l'époux est un riche marchand de bonne réputation. L'amant est un clerc qui remplit la fonction d'écrivain public. Lorsque ce dernier constate la méprise qui est la source du récit, il constate dédaigneusement:

Ainz sont les braies au vilain ${ }^{22}$.

Le mari est en revanche toujours désigné comme «li borgois» dans le reste du texte. Il n'est pas nécessaire que la femme soit infidèle, il suffit que l'époux paraisse tyrannique. Tel est le cas dans Les Trois Boçus où l'homme est un bourgeois dont la demeure est confortable et sa femme ne manque de rien. Mais l'époux est jaloux sans raison et, de plus, il a le tort d'être bossu... ${ }^{23}$

Le terme de vilain peut donc servir d'injure dans un contexte social assez éloigné du monde rustique. Toutefois les tabous essentiels sont respectés: le chevalier, même brutal, le prêtre, même lubrique, ne sont pas qualifiés de vilains.

Lorsque la scène se déroule au sein de la paysannerie, on peut observer le glissement de sens entre le nom de métier et l'injure. Le conte Les Quatre Sohais saint Martin commence ainsi :

Un vilain ot en Normandie ${ }^{24}$.

Ce paysan consciencieux et pieux ne manque pas de prier saint Martin avant de se mettre au travail. C'est pourquoi le saint un jour lui apparaît et le félicite:

Vilain, dist il, tu m'as mout chier

et il lui annonce qu'il peut laisser là sa herse et rentrer à la maison, car il lui accorde quatre souhaits. Arrivant chez lui plus tôt que d'ordinaire, le paysan est mal reçu:

Sa fame, qui chauçoit les braies

$\mathrm{Li}$ a dit «vilains, mal jor aies!» (v. 36-37)

Elle lui reproche sa paresse en une vigoureuse tirade. L'homme répond patiemment :

Tais, bele suer, ne te deshaite,

22. N.R.C.F., III, 17, v. 218.

23. N.R.C.F., $\mathrm{V}, 47$.

24. N.R.C.F., IV, 31, v. 1. 
Dist li vilains, que riches somes. (v. 46-47)

Il lui explique la chance qu'ils ont. La virago s'adoucit, court l'embrasser et l'appelle «mes amis, biaus dolz amis»... Le mari hésite à lui laisser formuler un des vœux :

Fames ont mout foles pansees.

Tost demanderiez fusees

De chanvre o de laine o de lin. (v. 69-71)

Il a le tort de se laisser fléchir. Par la faute de sa femme, le cadeau du bon saint Martin sera perdu. Consternés tous deux, ils reconnaissent leur erreur. Le paysan, jusqu'alors appelé «li vilains» par le conteur, est désigné comme «li prodom» dans la conclusion, et son épouse l'appelle poliment «sires » (v. 188).

Les conteurs, comme on le voit, savent habilement user de la polysémie du terme «vilain ». Le contexte indique s'il faut donner au mot un sens insultant.

Il est une autre raison, plus connue, qui peut dissuader le poète d'appeler vilain un paysan à qui il donne un rôle honorable: la traditionnelle association d'idée entre vilain et vilenie.

Contre elle, les protestations ne manquent pas, comme chacun le sait. Ainsi le Roman de la Rose affirme avec vigueur:

Je respons que nus n'est gentis

S'il n'est a vertuz antantis

Ne n'est vilains fors por ses vices,

Dont il pert outrageus et nices. (v. 18585-18588)

Nul n'est vilain s'il ne fait ou dit vilenie... Cet excellent principe est répété à satiété. On le trouve même dans le plus ordurier des textes, le Respit del curteis et del vilain, comme si l'auteur cherchait à se faire pardonner in fine ${ }^{25}$. Mais, dans la pratique, vilain et vilenie restent souvent associés. Ils le sont d'autant mieux que les recueils de proverbes colportent, sous une forme facile à mémoriser, les vices imputés au vilain. Les Proverbes au Vilain, composés entre 1171 et 1181 , connurent un succès immédiat et des imitations nombreuses ${ }^{26}$. Ils ont contribué à répandre dans le public les sentences qui accablent «le vilain», le classique repoussoir des héros preux et courtois lancé par l'épopée et le roman. Par exemple, les Proverbes ruraux et vulgaux rassemblent les formules les plus

25. D. Behrens, «Li respit del curteis et del vilain», Zeitschrift für französische Sprache und Literatur, 13 (1891), p. 154-158.

26. Li Proverbe au Vilain, die Sprichwörter des gemeinen Mannes, altfranzösische Dichtung nach den bisher bekannten Handschriften, éd. A. TöBLER, Leipzig, 1895. 
connues ${ }^{27}$. Le proverbe affirme catégoriquement et sans nuance, c'est une loi du genre, haussant le préjugé au rang de l'évidence.

Vilains toudis porquiert a brisser gentillesse. ( $\left.\mathrm{n}^{\circ} 232\right)$

Oingniez le vilain la paume et il vous chiera ens. $\left(n^{\circ} 311\right)$

Faites le bien a vilain et il vous fera mal. (nº 319)

C'est associer brutalement le personnage à la scatologie. Car vilenie est un terme polysémique lui aussi. Les fabliaux en usent souvent au sens le plus concret, témoin le conte de Rutebeuf intitulé De Charlot le Juif qui chia en la pel dou lievre:

Por li rendre la felonie

Fist en la pel la vilonie

(Vos saveiz bien ce que vuet dire) ${ }^{28}$.

D'autres contes mettent en scène des vilains sous un titre suffisamment évocateur, tel De la crote.

Fort heureusement, les poètes ne sont pas prisonniers du vocabulaire. L'auteur qui se refuse à ternir l'image d'un personnage, celui qui veut présenter un paysan sous un jour favorable peut éviter de l'appeler vilain sans user non plus du mot «païsant» alors peu répandu. Les plus habiles connaissent plusieurs façons de se tirer d'embarras.

Jean Bodel a donné l'exemple dans un conte où, contrairement à ses habitudes, il n'emploie pas une seule fois le mot «vilain». Il met en scène dans Barat et Haimet deux larrons dont les prouesses stupéfient leur compagnon Travers, qui pensait apprendre le métier en leur compagnie. Se sentant incapable de les égaler, il les quitte et rentre au village où l'attend sa femme, et il se met au travail.

Mout devint saiges hons et boens,

Et mout volentiers gaaigna;

Et tant conquist et amassa

Qu'il ot assez et un et el.

Un bacon fist devant Noel

D'un porc qu'il ot en sa maison

Encraissi tote la saison ${ }^{29}$.

27. J. ULRICH, «Proverbes ruraux et vulgaux », Zeitschrift für französische Sprache und Literatur, 24 (1902), p. 1-35.

28. Rutebeuf, t. II, p. 270, v. 113-115.

29. N.R.C.F., II, 6, v. 119-124. 
L'auteur ne dit nulle part quel est le métier de Travers, mais on comprend sans peine qu'il est paysan. Un jour où il est allé en forêt chercher des fagots, surviennent les deux larrons, qui entrent dans la maison, s'assoient, conversent avec la dame, et scrutent les coins et recoins de la demeure. Ils voient la charpente et la pièce de lard pendue entre deux traverses. Après leur départ, Travers rentrant chez lui comprend vite qu'on en veut à son bacon. Il le dépend et le cache sous un coffre renversé. Arrive la nuit, qui sera fort agitée. La maison comporte deux pièces, la salle et la chambre. Elle est dans un enclos qui abrite aussi l'étable où se trouve la vache. Quand le feu «de bûche et de charbon» est éteint, les deux larrons reviennent et s'introduisent en perçant un trou dans le mur de torchis. Le bacon est pris, repris et reperdu au cours de poursuites échevelées dignes d'un vaudeville. Pour finir, le bacon est mis à cuire dans la chaudière qui pend au-dessus du feu au moyen d'une «hardière» (corde munie d'un crochet), et partagé entre le couple et les deux voleurs. Car ceux-ci avaient découvert le toit au-dessus du feu et pêchaient les tranches de lard avec une tige épointée... Travers n'a trouvé d'autre moyen de se débarrasser d'eux que de céder une partie de son bien. Le héros de l'histoire est toujours appelé par son nom, Travers. Sa femme l'appelle «sire Travers», et lui l'appelle «bele suer» ou Marie.

Que le paysan soit nommé ou pas, un autre procédé courant consiste à ne mettre le mot «vilain» que dans la bouche de ses adversaires. C'est le cas dans Constant du Hamel, écrit dans la première moitié du XIII ${ }^{\mathrm{e}}$ siècle dans la zone picardisante:

Ma paine metrai et ma cure

A raconter une aventure

De sire Costanz du Hamel ${ }^{30}$.

La femme de ce paysan est belle, trop belle pour lui, pense le prévôt:

Ha, dame ! fait il, mout me poise

Que teus vilains vos a en garde. (v. 45-46)

Le forestier et le curé pensent de même et tentent eux aussi de séduire dame Ysabeau. Celle-ci parlant de son époux emploie les mots «mon seignor», «mes sires ». Les deux ministériaux tentent de la faire céder en menaçant de ruiner son mari, qu'ils traitent de «dant vilain de pute foi».

Autre exemple, Le Povre Cler, qui conte l'histoire d'un jeune étudiant:

Que a Paris ot demoré

Uns clers, tant que par povreté

Li covint la vile a laissier

30. N.R.C.F., I, 2, v. 1-3. 
Et il n'ot mais que engagier

Ne que vandre dont rien aüst.

... Miauz valt il laissier son aprandre ${ }^{31}$.

De quel pays est-il, on ne sait, peut-être de «l'ouest du domaine d'oil» où fut composé le conte. Le jeune clerc sans un sou se met bravement en route à pied, mais ses forces le trahissent et il doit s'arrêter dans un village où il ne connaît personne. Déconvenue que dut subir sans doute maint fils de paysan pauvre, évoquée par Rutebeuf (cf. l'introduction).

... en une vile qu'il trova

S'an est chiés un vilain entrez. (v. 20-21)

La dame refuse de le recevoir. Trop fatigué pour se remettre en route, le clerc erre autour de la maison jusqu'au moment où arrive le maître de celle-ci, «uns prodom», qui l'interroge, le fait entrer et l'héberge le mieux possible malgré sa femme qui comptait recevoir le prêtre son amant. Elle avait déjà tout préparé pour lui... Le paysan revient du moulin:

Si port farine de froment

Por faire a mes enfanz do pain. (v. 83-84)

Grâce à l'ingéniosité du clerc, le maître de maison fait profiter son hôte des bonnes choses préparées pour le prêtre, que l'on expulse vigoureusement.

Le sens de l'hospitalité dont fait preuve ce paysan est dans les contes une vertu majeure: jongleurs et trouvères en ont souvent besoin dans la pratique et dans l'univers des fabliaux. Les poètes se vengent de ceux qui refusent d'accueillir autrui en inventant diverses manières de les punir. Parmi ces mauvaises gens, des prêtres, un meunier, mais aucun paysan...

L'auteur anonyme du Povre Cler tire la morale de son conte en puisant dans les proverbes:

Cist fabliaus nos dit et raconte

Q'an son respit dit li vilains

Que a celui doint l'an del pain

Q'an ne cuide jamais revoir. (v. 242-245)

Malgré l'allusion flatteuse au vilain en tant que dépositaire de la sagesse des nations, le poète évite d'employer le mot pour désigner un paysan si hospitalier. 


\section{«Ni tout à fait bon, ni tout à fait méchant»}

Les poètes bienveillants n'installent pas sur scène un personnage qui ferait du paysan l'exact contraire du «Caliban médiéval». Celui-ci ne disparaît pas, il servira longtemps à faire rire, et les paysans les plus honorables lui empruntent éventuellement quelques traits. Aux travers du vilain repoussoir s'ajoutent des caractères favorables qui améliorent le tableau d'ensemble comme vont le montrer quelques exemples.

Le vilain repoussoir est sale et le conte du Vilain Asnier a valeur de symbole. Le personnage accoutumé à conduire deux ânes chargés de fumier tombe en syncope lorsqu'il s'aventure dans les étalages des marchands d'épices. Pour le ranimer, il suffit de lui mettre sous le nez une fourchée de fumier. Plus souvent évoquée que cette familiarité avec toute forme d'ordure est la négligence des soins corporels. Le paysan ne suit pas les modes masculines, qu'il soit sympathique ou non. Dans Le Vilain au bufet, les travers moraux du vilain classique sont attribués au sénéchal qui est avare, jaloux de toute faveur accordée à autrui, malpoli et, faute impardonnable évidemment :

Les menestreus haoit a mort ${ }^{32}$.

Le vilain Raoul, héros de l'histoire, est en revanche un brave homme tout heureux de profiter de la générosité du comte. Ce jour-là, en effet, qui veut entrer au château entre et peut manger et boire tant qu'il lui plaît. Raoul fait comme tout le monde. Mais le poète n'a pas flatté son aspect extérieur:

Deslavés iert, s'ot chief locu

Que bien ot quarante ans vescu

Qu'il n'avoit eü coiffe en teste. (v. 97-99)

Il est fort mal reçu par le sénéchal qui le soufflette et lui dit, en jouant sur le mot «buffet» qui peut vouloir dire «dressoir» ou «gifle»:

Or sié, fait il, seur ce bufet

Que je te prest pour seoir sus! (v. 132-133)

Raoul ne s'émeut pas, mais à la fin du repas, il vient rendre avec usure son «bufet» au sénéchal.

Et li vilains la paume estant

Qu'il ot dure et plaine de jales

N'a si fort home jusque en Gales. (v. 172-174) 
L'aspect peu soigné va de pair en effet avec une force redoutable. Constant du Hamel, dit le prévôt, est:

Gros et malostruz

Il n'est ne res ne tonduz

Ainz est hideus et deslavez ${ }^{33}$.

Mais guidé par son astucieuse épouse qui organise la vengeance, Constant se débarrasse seul de ses trois persécuteurs et leur fait crier grâce.

Le malin jongleur Boivin, lorsqu'il se déguise en paysan, revêt un costume de bureau gris et de gros souliers de cuir de vache. Mais cela ne saurait suffire puisque le vilain est hirsute:

Un mois et plus estoit remese

Sa barbe qu'ele ne fust rese ${ }^{34}$.

Les conteurs cependant se dispensent dans la majorité des cas de décrire l'allure du paysan. L'action suffit, comme il se doit dans le récit bref.

Pauvre ou à son aise, le paysan des contes consomme des aliments simples, monotones et peu relevés par le mode de préparation. Choux, raves, céréales, tout cuit dans une marmite. Telle est la bouillie de lait caillé et d'œufs, «li matons », du Vilain de Bailluel, ou le «morteruel» du Vilain de Farbu. Le bacon est un régal convoité au centre de plusieurs contes où il passe de main en main comme dans Le Sacristain. Même au banquet de mariage dans Jouglet, le menu reste le même. Aussi celui qui, par une chance extraordinaire, trouve deux perdrix le long de la haie est-il tout excité à l'idée du festin qu'il va savourer avec sa femme:

Or ça mon bon hanap de madre

Et ma plus blanche nape!

Si l'estenderai sus ma chape,

Souz cele treille en cel prael ${ }^{35}$.

La viande rôtie du chevalier et du bourgeois suscite l'envie chez le vilain: «Il dit, s'il voit venir I gentil home qui porte un esprivier sus son poig: Ha! fait il, cil huas mangera enquenuit une geline et mi enfant en fuissent tuit saoul!» Tel est le «vilains chenins ${ }^{36}$.

L'envie ne se borne pas à la nourriture. «Rabaisser gentillesse», comme dit le proverbe, est le sentiment attribué systématiquement aux vilains dans

33. N.R.C.F., I, 2, v. 52-54.

34. N.R.C.F., II, 7, v. 13-14.

35. N.R.C.F., IV, 21, v. 68-71.

36. E. FARAL, «Des vilains...», p. 251. 
les textes satiriques qui passent en revue les divers «estats du monde». Ainsi s'exprime la crainte qu'inspire l' «autre», perçu comme une menace. En revanche les fabliaux ne construisent pas de contes sur ce thème. Chevaliers et paysans figurent dans des récits différents, si bien que les paysans n'ont affaire qu'à des ministériaux comme dans Constant du Hamel.

Il en va tout autrement de l'animosité à l'égard du clergé. Entre celui-ci et la paysannerie, la haine est réciproque, affirme le texte Des XXIII manières de vilains cité précédemment:

Por ce que li cler me soutiennent

Et me revestent et retiennent

Por ce he je tous les vilains

Qui n'aiment clers ne chapelains ${ }^{37}$.

On sait que le prêtre de village, qui courtise ses paroissiennes au grand dam de ses paroissiens, est le héros de nombreux contes. Héros parfois malheureux : le prêtre est accablé d'injures et de coups, émasculé, couvert de honte devant les villageois. Jamais aucun paysan n'est dans les contes maltraité aussi sauvagement. Certes il est parfois cocu, mais c'est un malheur qu'il a en commun avec les autres hommes, sans oublier les chevaliers. Le châtiment réservé au prêtre est en général public, ce qui en accroît la dureté.

Il n'y a donc pas de frontière étanche entre le vilain repoussoir et le paysan honorable, certains traits étant communs aux deux. Comme nous allons le voir, si l'image du paysan sort grandie du corpus des fabliaux et de textes divers qui leur sont contemporains, c'est avant tout parce que les auteurs lui attribuent une place définie dans la société, la place de chef de feu, comme le sont les artisans, les marchands et le seigneur lui-même.

\section{Le chef de feu et la maîtresse de maison}

Le chef de feu est seul pris en compte, sauf exception, dans les chartes de franchises, les accensements collectifs, les premiers recensements. C'est la situation de la plupart des paysans des fabliaux : un homme établi, «chasé », qui possède une demeure, des terres qu'il met en valeur comme il le juge bon, qui habite une maison avec femme et enfants, et souvent un ou plusieurs domestiques. Il fait partie d'une communauté paroissiale.

Grande ou petite, son exploitation correspond à ce que Beaumanoir appelle «tenure en vilenage: Nous apelons vilenage eritage qui est tenu de 
seigneur a cens ou a rente ou a champart, car de celi qui est tenu en fief, l'en ne doit nule tel redevance ${ }^{38} »$.

On ne voit point le paysan des contes recevoir des directives de quiconque. La seule allusion faite au métayage se trouve dans la seconde version du Sacristain, composée dans le centre de la France: «Sire Tibout le moitoier» cultive les terres d'une abbaye ${ }^{39}$.

Le travail principal est pour tous le labour. La charrue a dans les contes une valeur de symbole. Lorsque le Vilain Mire rentre chez lui comblé d'honneur et de richesses par le roi, il est dit de lui :

N'onques puis ne fu a charue ${ }^{40}$.

Posséder une charrue et des bêtes de trait est essentiel et distingue le gagne-petit de l'homme à son aise. Le vilain Liétard du Roman de Renart tient quatre bœufs qui lui permettent de cultiver son bien, qu'il n'a cessé d'agrandir. L'un d'eux, doté de la parole comme Renart et Ysengrin, joue un rôle important dans l'histoire. Une dizaine de proverbes mentionnent le bœuf, l'animal le plus puissant, le plus coûteux et le plus long à dresser. En revanche, un seul, Mettre la charrue avant les bœufs, nomme l'instrument aratoire. Chaque genre littéraire a ses traditions et sa mythologie.

Un paysan riche comme l'est Aloul emploie à demeure plusieurs bouviers pour conduire son train d'attelage. Leur force, leur lenteur et leur formidable appétit compliquent les péripéties nocturnes du fabliau.

Le paysan des fabliaux a toujours des bêtes, ne serait-ce qu'une vache. Cultivateur et éleveur en même temps, unissant Caïn et Abel, ses tâches sont diverses. Étienne de Fougères (mort en 1178) le savait bien: sur trente strophes consacrées aux vilains, deux énumèrent leurs travaux ${ }^{41}$. Les contes montrent eux aussi le paysan toujours occupé. «Li prodom», dont la fille est si capricieuse qu'il ne peut garder aucun valet, se plaint de n'avoir personne pour l'aider.

$S$ 'an aüst il mout grant mestier

A ses blez batre et a vener

Et a sa charue mener

Et a faire s'autre besoigne ${ }^{42}$.

Le jour où un valet plus futé que les autres se présente:

Li vilains estoit en la cort

38. Philippe de Beaumanoir, Coutumes de Beauvaisis, éd. A. Salmon, t. 1, Paris, 1970, no 467.

39. N.R.C.F., VII.

40. N.R.C.F., II.

41. ÉtIENne de Fougères, Le Livre des manières, éd. R. A. Lodge, Genève, 1979.

42. N.R.C.F., IV, 26, v. 26-29. 
Ses bestes atire et atorne

Et sa buche au soleil retorne ${ }^{43}$.

Le jeune homme explique qu'il connaît le métier:

Que bien sait arer er semer

Et bien batre et bien vaner

Et tout ce que vallez doit faire ${ }^{44}$.

Cependant, nul conte n'est aussi bavard et détaillé sur les travaux à la ferme que les deux plus anciens poèmes sur les biens d'un ménage. L'Oustillement au Vilain fait la liste impressionnante de tout ce qu'il faudrait posséder pour que la ferme fonctionne au mieux : instruments agricoles, ustensiles de cuisine, meubles, etc. Il donne en même temps de sages principes d'économie domestique: pour chaque tâche les outils et l'équipement adéquats, chaque travail accompli au bon moment, un emplacement pour chaque objet, rien n'est oublié ${ }^{45}$. Il faut tirer parti de tout, ne rien gaspiller, boire plus d'eau que de vin. L'idéal n'est pas le luxe, mais une honnête aisance. Dans ce long poème du XIII ${ }^{\mathrm{e}}$ siècle, les humbles réalités de la vie paysanne ne sont ni laides ni ridicules, le travail est utile et bienfaisant. De plus, il est guidé par une pensée rationnelle qui l'organise dans le temps et dans l'espace ${ }^{46}$.

Ce texte et le Dit de ménage, de peu postérieur, ont un double intérêt. D'une part, ils montrent des clercs, des hommes instruits capables de versifier, qui connaissent la campagne. Dans le second texte, daté du tout début du XIV siècle, un des interlocuteurs avoue sans embarras ses origines paysannes, ce qui est fort rare :

- Je sai bien qu'il leur faut, né fui en un hamel.

Il leur convient par force la fourche et le flael,

Une piaule et j pic, la houe et le hoel

Le rouable et la fauz, la queue et le martel. (v. 133-135)

D'autre part, ces poèmes, par le biais d'une liste d'objets, font l'apologie du mariage-établissement, considéré comme la norme. Seul un insensé se marie sans avoir de quoi entretenir une famille... La malheureuse histoire du Vallet qui d'aise

43. Ibid., v. 50-51.

44. Ibid., v. 65-67.

45. U. Nystrom, Poèmes français sur les biens d'un ménage depuis L'Oustillement au Vilain du XIII siècle jusqu'aux Controverses de Gratien du Pont, Helsinki, 1940.

46. M.-T. Lorcin, «De l'Oustillement au Vilain ou l'inventaire sans raton laveur», Revue historique, 274 (1973), p. 321-339. 
a malaise se met est l'illustration de ce principe ${ }^{47}$. Principe qui inspire également la déclaration que fait le jeune Gauthier dans Le Jeu de Robin et Marion:

Mais j'ai au mains ronchi traiant

Boin harnas et herche et carue,

Et si sui sires de no rue.

S'ai houche et sercot tout d'un drap;

Et s'a me mere un boin hanap

Qui m'eskerra s'ele mouroit,

Et une rente c'on li doit

De grain seur un molin a vent,

Et une vake qui nous rent

Le jour assés lait et froumage;

N'a il en moi boin mariage,

Ddites, Perrete ? $^{48}$

J'ai montré ailleurs le rôle capital joué par la maîtresse de maison dans les fabliaux ${ }^{49}$. Bourgeoise ou paysanne, elle dirige les êtres et les choses qu'abrite la demeure. Elle tient les clés des locaux et des coffres, elle conserve les denrées et en règle l'usage, elle distribue la nourriture à toute la «mesnie». Elle commande aux servantes qui, comme elle, restent à la maison.

Seules les femmes maîtrisent le feu domestique. Si l'homme, maître du feu de la forge, doit allumer celui de la maison, il s'époumone en vain et pleure à cause de la fumée ${ }^{50}$.

Dame et servante ont en effet partie liée et cette coalition est presque imbattable. Dans Aloul, le riche paysan et ses bouviers pourchassent dans l'obscurité le prêtre amant de la dame qui s'est introduit dans la demeure. La vieille servante Hersent apostrophe les bouviers qu'elle traite de «vilains » et de «filz a putain» parce qu'ils veulent faire honte à leur dame, qui est «mout bone dame».

Bon renon a de preude fame

Et vous li fetes tel anui ${ }^{51}$ !

Sa furieuse tirade de vingt-cinq vers contient une menace à prendre au sérieux. Si vous fâchez ma dame, elle vous supprimera les œufs et le fromage.

47. N.R.C.F., VIII, 100.

48. Adam le Bossu, Le Jeu de Robin et Marion, éd. E. Langlois, Paris, 1992, v. 639-650.

49. M.-T. Lorcin, «La femme est empereur en son hôtel, ou la maîtresse de maison dans les fabliaux », dans A. Bleton-Rugert, M. Pacaut et M. Rubellin éd., Georges Duby, regards croisés sur l'œuvre, femmes et féodalité, 2000, p. 213-218.

50. M.-T. LorCin, «Le feu apprivoisé: l'homme, la femme et le feu dans les fabliaux », Revue historique, 268 (1982), p. 3-15.

51. N.R.C.F., III, 14, v. 397-399. 
Des pois mengerez et du pain!

Les bouviers inquiets se dépêchent de s'excuser et, malgré la colère d'Aloul, ils abandonnent la poursuite et retournent se coucher.

Le couple de Constant du Hamel et sa femme est en revanche uni. Aussi résiste-t-il aux agressions extérieures. Ysabeau invente le moyen de riposter et le couple le met à exécution avec l'aide de la fidèle servante.

Dans des circonstances moins dramatiques, les contes montrent paysan et paysanne discutant des problèmes de la vie quotidienne. Vaut-il mieux faire battre l'avoine qui est sous l'auvent et la vendre pour pouvoir rembourser ce que nous devons au voisin? Cela permettrait aussi de nourrir les bêtes. La cour est vide, il n'y a plus ni paille ni fanes de pois. Ou vaut-il mieux vendre celle qui est en grains dans le grenier ${ }^{52}$ ? Les conversations entre époux sont riches de détails sur les travaux et les jours. Il est temps d'aller au moulin, nous n'avons plus que deux pains, dit une femme à son mari. Impossible, répond l'homme, je suis malade depuis deux jours. Réveillez plutôt Martin, ce colporteur qui couche chez nous comme il le fait souvent. Mais Martin regimbe. Quand vous avez tué le cochon, vous ne m'avez fait goûter ni boudin, ni os. Les autres du village me traitent mieux que vous ne le faites. Allons, dit la dame, ne nous chamaillons pas. Si je te fais griller au feu une belle tranche de bacon pour manger avec du pain, ne voudras-tu pas faire ce que je te demande? Martin réjoui accepte et la dame presse de nouveau son mari de se lever pour qu'il aille couper une tranche de lard afin de faire à Martin une «carbonnée ». Le paysan grimpe à l'échelle et atteint le garde-manger. De quel côté veux-tu que je le taille? Cela m'est égal, dit Martin, vous connaissez votre bacon mieux que moi. Tous ignorent encore que le bacon a été dérobé pendant la nuit ${ }^{53} \ldots$

Le sens pratique et la dextérité du paysan qui sait tirer profit de tout deviennent un sujet bouffon dans un poème du XIII ${ }^{\mathrm{e}}$ siècle intitulé $L^{\prime}$ Eschacier ${ }^{54}$.

Le conteur rencontre en se promenant un vilain bien vêtu mais avec une jambe de bois, qui répond aimablement à son salut. Tous deux cheminent côte à côte plus d'une lieue en bavardant. Interrogé sur son malheureux accident, l'«eschacier» explique que cela lui est arrivé deux ans et demi auparavant, mais qu'il a su s'en accommoder au mieux. Avoir un seul pied à chausser est économique : les souliers coûtent cher et durent moins longtemps qu'un pilon. De plus il ne craint plus de ce côté ni de se cogner, ni de marcher sur des épines.,

52. «Le prestre comporté», N.R.C.F., IX, 102.

53. «Le Sacristain», seconde version, N.R.C.F., VII, 74.

54. A. Jubinal, Jongleurs et trouvères...des XIII et XIV siècles, Paris, 1835, rééd. Genève, 1977 , p. $158-163$. 
S'il marche sur serpent

De l'envenimement

Ne li estiet douter,

Que il ne puet grever.

S'il marche sur chardon

N'en donroit un bouton. (v. 95-100)

En outre, cette jambe de bois, s'il la délace, se révèle utile:

Si puet-il de l'eschace

S'aillie pesteler

Et son poivre souder

Et son commin broier,

Et son feu atisier;

Et puet ses nois brisier,

Et son huis chevillier. (v. 97-103)

Il peut aussi s'en servir pour tuer le porc ou le chien et battre sa femme. C'est l'outil à tout faire. Au reste, on trouve sur les tas d'ordures maint vieux soulier, mais on ne trouve jamais une «eschace» qui, si elle est bien ferrée, dure très longtemps. Le jongleur, comme il se doit, tire la leçon de l'histoire:

Por ce di que fols est

Vilains qui a ii piez

Que l'un ne soit trenchiez. (v. 144-146)

Le paysan chef de feu et son épouse se distinguent nettement de la troupe anonyme des valets et «baiasses», des bouviers et pasteurs qui n'ont ni tenure ni famille, encore plus des ribauds que l'on embauche à l'occasion pour transporter quelque fardeau. Les jeunes valets à la recherche d'un travail ont dans les contes le même statut que les clercs désargentés qui demandent l'hospitalité, ou que le jeune chevalier qui fait de même après avoir perdu son équipement dans les tournois. Si le clerc ou le valet couche avec la fille ou l'épouse de son hôte, l'un et l'autre se tirent indemnes de l'aventure ${ }^{55}$. Il en est de même s'ils volent un mouton ${ }^{56}$. Les jeunes hommes sont sans attache, sans responsabilité et sans scrupule.

La jeune paysanne non mariée n'a pas de rôle caractérisé dans les contes, contrairement à la maîtresse de maison. Elle est, comme toutes les jeunes filles des fabliaux, trop naïve ou trop perverse. En revanche, dans Le Jeu de Robin et Marion, son rôle est de civiliser l'homme. Marion reproche aux jeunes paysans mal dégrossis leur goût pour les jeux grossiers et leur manque d'égard envers

55. «Gombert et les deus clers», N.R.C.F., IV, 35.

56. «Estula», N.R.C.F., IV, 38. 
elle et envers ses compagnes. D'un genre littéraire à l'autre, les préférences et les tabous changent.

Tous les genres en revanche sont d'accord sur un point: le métier de paysan n'est pas le plus décrié. Il suffit pour s'en convaincre de lire les moqueries lancées dans Les Deus Bourdeurs ribauds et autres pièces similaires: bouvier, meneur d'aveugle, charretier, larron promis au pilori, et enfin cureur de fosses ${ }^{57} \ldots$ Une parodie des chartes de franchises, Le Privilege aus Bretons, brode longuement sur ce que revendiquent les Bretons de Paris devant le roi Philippe. Le roi consent volontiers à confirmer cette «franchis » qui ne lui coûte rien au profit d'immigrés récents pleins de bonne volonté:

De balais fer, de curer fos ;

Bien fu en parchemin enclos

Et coper au bois la genes,

Et porter a la vil grant fés,

Et fiens porter en la chiviere,

Breton devant, Breton derriere;

Et eus et toute la compagne,

Doivent reperier en longaigne:

Tout ce fist il confermer ${ }^{58}$.

Les éléments positifs accolés au classique stéréotype sont, comme on le voit, de nature morale et sociale. Que le paysan reste hirsute n'a pas d'importance: le conteur ne se croit pas obligé de le décrire. Ce qui compte, ce sont le statut social et les qualités morales qui peuvent le faire estimer par ses contemporains.

L'historien est connu pour chercher à mettre au jour une chronologie, ou tout au moins une évolution. De chronologie, il ne saurait être question ici car les sources s'y prêtent mal. On est trop heureux si tel conte peut être daté de la première ou de la seconde moitié du XIII ${ }^{\mathrm{e}}$ siècle... Une évolution, en revanche, paraît sûre.

À l'orée du XIV e siècle existe dans les textes en langue d'oïl un personnage que l'on connaissait peu un siècle auparavant. Il s'agit du paysan chef de feu, qui exploite ses terres avec compétence, qui a des aventures et mésaventures proches de celles que connaissent les artisans, bourgeois et même chevaliers dans leurs propres cadres de vie. Les écrivains le désignent de diverses manières: vilain, païsant, preudon...

57. E. FARAL, Mimes français, p. 83-11.

58. Ibid., p. 28. 
On pourrait craindre que l'image du paysan ne change pas puisque certains auteurs du XIII ${ }^{\mathrm{e}}$ siècle continuent de l'appeler «vilain», un terme qui sert aussi d'apostrophe injurieuse. Mais l'analogie «vilain»= paysan, qui allait de soi au temps où écrivait Jean Bodel (1190-1200 environ), ne l'est plus au temps de Rutebeuf. Cet artiste du mot n'emploie pas au hasard les différents termes existants pour désigner un paysan.

Rutebeuf se vante de n'être pas «ovriers de mains » et l'on ignore tout de ses origines. C'est en son temps le plus parisien des poètes. Amateur de potins sur la cour royale et sur la tenue des couvents, il prend parti avec fougue dans les querelles qui secouent le milieu intellectuel de la Chrétienté, il compose sur commande des poèmes de propagande sur la politique extérieure de la France en Italie et en Orient. Il connaît la capitale des rois capétiens à fond et ne la quitte jamais, semble-t-il. Il pourrait écrire un guide sur les églises et autres établissements religieux à l'usage des visiteurs... Pourtant il montre à diverses reprises qu'il s'intéresse aussi à la vie rurale. Il célèbre l'arrivée du printemps ainsi : après être restée cachée tout l'hiver...

... de souz terre ist la vermine ${ }^{59}$.

Les arbres se couvrent de feuilles, la terre se pare de fleurs multicolores. L'homme sort enfin de chez lui, et pour travailler:

Li preudons, quant voit le jor nei,

Reva areir en son jornei.

Apres areir, son jornei samme ${ }^{60}$.

Le poète aurait pu choisir d'autres topoi montrant les riches oisifs partir à la chasse ou se promener avec des dames.

Rutebeuf emploie deux fois le mot «païsant». Le premier passage, déjà cité, concerne le paysan pauvre qui se prive pour envoyer son fils étudier. La seconde occurrence se trouve dans l'Estat du monde: «ces païsanz des vignes » qui demandent salaire ${ }^{61}$. Il fait allusion aussi à la foule des petites gens, composée d'illettrés à qui les prédicateurs font croire ce qu'ils veulent:

Je ne blame pas gent menue:

Il sont aussi com beste mue.

L'en lor fet can'on veut acroire ${ }^{62}$.

59. «La voie d'humilité»: Rutebeuf, t. 1, v. 2.

60. Ibid., 9-11.

61. Ibid., t. 1, v. 144-146.

62. «De sainte Église», ibid., t. 1, 97-100. 
Dans ces passages traitant du monde réel qui l'entoure, Rutebeuf n'utilise jamais le mot «vilain ». Il le réserve pour un contexte très différent. Par exemple il glisse une allusion aux proverbes:

Li vilains dist: tost vont noveles ${ }^{63}$.

Il s'étend davantage dans Le Miracle de Théophile. Le héros, devenu serviteur de Satan auquel il a vendu son âme d'intellectuel déçu, se promet de traiter sans pitié ceux qui sont devenus ses corvéables. Rutebeuf met dans sa bouche le parler méprisant du mauvais seigneur:

Li vilain por moi aorer

Et je les ferai laborer.

... je lor serai fel et irous ${ }^{64}$.

La pièce de théâtre plonge le lecteur-auditeur dans la fiction de l'au-delà. Le fabliau Le Pet au Vilain y baigne encore plus résolument puisque l'histoire se déroule pour l'essentiel en enfer au milieu des conversations des diables. Les poncifs traditionnels à l'égard du vilain servent tous d'arguments ici: ordure, mauvaise odeur, ni foi ni loyauté, haine envers prêtres et clercs. Le vilain ne peut trouver place ni en paradis ni en enfer. La conclusion absurde du débat place le récit dans l'irréel et dans l'énorme bouffonnerie que nul n'est invité à prendre au sérieux.

Artiste du vocabulaire, Rutebeuf réserve le mot «vilain » au folklore et au monde des extraterrestres. Les paysans ses contemporains sont des «païsants » ou des «preudons».

Au-delà des mots, l'idée semble généralement admise dans le courant du XIII ${ }^{e}$ siècle d'un dédoublement d'image. Le vilain repoussoir et ridicule ne disparaît pas: toute société cultive ses boucs émissaires, ses têtes de Turc, ses clowns. Mais la littérature de divertissement offre aussi le portrait d'un paysan plus digne. Pendant que ses pareils obtiennent d'introduire des mots de leur parler dans les chartes de franchises et autres documents de la pratique, les poètes lui font une place à côté de l'artisan et du curé, du marchand et du chevalier, qui tous sont également des chefs de feu.

Marie-Thérèse LoRcIN - Université Lumière Lyon 2-UMR 5648 (CIHAM), 18, quai Claude Bernard, 69365 Lyon CEDEX 07

63. «Complainte du comte de Poitiers», ibid., t. 2, v. 123.

64. «Le miracle de Théophile», ibid., t. 2, v. 332-336. 


\section{Du vilain au paysan sur la scène littéraire du XIII ${ }^{\mathrm{e}}$ siècle}

Le stéréotype du vilain, incarnation de la bassesse, ne disparaît pas, perpétué par les proverbes et les textes comiques qui illustrent aussi les stéréotypes de la femme et du prêtre. Mais apparaît un autre personnage, un paysan réhabilité, humanisé, même s'il est encore parfois appelé «vilain», le mot «païsant» étant encore très rare. Les poètes, dont beaucoup sont sans doute d'origine rurale, le montrent hospitalier, patient, voire instruit. Il n'est pas l'objet comme l'est le prêtre de la vindicte publique, ni ne subit de châtiment dégradant. Il exploite ses terres et gère son domaine normalement. En tant que chef de feu ayant ses responsabilités et son autonomie, il occupe une place déterminée dans la société, tout comme l'artisan, le commerçant et le seigneur. À côté du Caliban médiéval existe le paysan, celui dont le poids et parfois le vocabulaire apparaissent à la même époque dans les chartes de franchises.

France - paysan - littérature - vocabulaire - Rutebeuf - fabliaux - chef de feu.

\section{From Villein to Peasant in the Literature of the 13th Century}

The stereotype of the churl («vilain» or «villanus»), embodiment of baseness, does not disappear in the thirteenth century: on the contrary, it is immortalized by the proverbs and the comic literature which also illustrate the stereotypes of the woman and the priest. But another character appears, a rehabilitated and humanized farmer, even if he is still called sometimes villanus, the word «païsant» (peasant) being yet very rare. The poets, among whom many were doubtless of rural origin, picture him as hospitable, patient, even educated. Unlike the priest, he his not the object of public condemnation and undergoes no degrading punishment. He runs his lands and manages his farm normally. As head of the household («chef de feu»), with his responsibilities and his autonomy, he occupies a specific position in the society, much as the craftsman, the merchant and the lord. Besides the medieval Caliban, the farmer does have a real existence, and contemporary charters of emancipation are the witnesses of his strength and sometimes of his own language.

France - peasant - literature - vocabulary - Rutebeuf - fabliaux - household. 